\title{
Mitochondrial DNA Mutations Associated with Neuromuscular Diseases: Analysis and Diagnosis Using the Polymerase Chain Reaction
}

\author{
DOUGLAS C. WALLACE, MARIE T. LOTT, ANGELA M. S. LEZZA, PETER SEIBEL, \\ ALEXANDER S. VOLJAVEC, AND JOHN M. SHOFFNER
}

Departments of Biochemistry [D.C.W., M.T.L., A.M.S.L., P.S.], Neurology [D.C.W., J.M.S.], and Nephrology [A.S.V.], Emory University School of Medicine, Atlanta, Georgia 30322, Dipartimento di Biochimica e Biologia Molecolare, Università di Bari, 70126 Bari, Italy [A.M.S.L.], and Fachbereich Chemie der Philipps-Universität, Marburg, Federal Republic of Germany [P.S.]

\begin{abstract}
A number of neuromuscular diseases are associated with molecular defects in the mitochondrial DNA (mtDNA). These include: 1 ) a missense mutation at nucleotide 11778 in the mtDNA of Leber's hereditary optic neuropathy patients; 2) a heterogeneous array of deletions in the mtDNA of ocular myopathy patients; and 3) small deletions and point mutations in the mtDNA of myoclonic epilepsy and ragged red fiber disease patients. We can now diagnose these diseases at the molecular level from small patient samples by amplifying the affected mtDNA regions using the polymerase chain reaction. Leber's hereditary optic neuropathy is diagnosed through loss of an SfaNI restriction site. Ocular myopathy deletions are identified by differential amplification across deletion breakpoints. Familial diseases such as myoclonic epilepsy and ragged red fiber disease might be diagnosed by identifying small deletions through amplification and electrophoretic analysis of the entire mtDNA genome or by identifying point mutations through differential oligonucleotide hybridization. As additional mtDNA molecular defects are identified, molecular analysis will likely become a primary tool for the diagnosis of these diseases. (Pediatr Res 28: 525528,1990 )
\end{abstract}

\section{Abbreviations}

mtDNA, mitochondrial DNA

PCR, polymerase chain reaction

RRF, ragged red fiber

MERRF, myoclonic epilepsy and ragged red fiber disease

MELAS, mitochondrial encephalomyopathy, lactic aci-

dosis, and stroke-like symptoms

KSS, Kearns-Sayre syndrome

CEOP, chronic external ophthalmoplegia plus nt, nucleotide

LHON, Leber's hereditary optic neuropathy

$\mathrm{O}_{\mathrm{II}}$, heavy strand origin

$\mathrm{O}_{\mathrm{L}}$, light strand origin

Several neuromuscular diseases have recently been associated with defects in the mtDNA (1-5). Specific DNA changes have been associated with $\operatorname{LHON}(6,7)$, the ocular myopathies, in-

Correspondence and reprint requests: Douglas C. Wallace, Ph.D., Emory University School of Medicine, Department of Biochemistry, Atlanta, GA 30322

Supported by NIH Grant NS21328-05 and Muscular Dystrophy Clinical Research grant awarded to D.C.W. cluding CEOP and KSS (8-14), and MERRF associated with stroke-like symptoms (15).

Each human cell has thousands of mtDNA (16), each coding for a complete set of ribosomal RNA and transfer RNA, as well as 13 polypeptides of oxidative phosphorylation. These polypeptides include seven subunits (ND1, 2, 3, 4, 4L, 5, and 6) of respiratory complex I (NADH-ubiquinol oxidoreductase), one subunit (cyt b) of respiratory complex III (ubiquinol-cytochrome c oxidoreductase), three subunits (COI, COII, and COIII) of complex IV (cytochrome c oxidase), and two subunits (ATP6 and 8) of complex V (ATP synthase).

The genetics of the human mtDNA abide by three unique principles. First, they are maternally inherited $(17,18)$. Second, mixed populations of mutant and wild-type mtDNA (heteroplasmy) segregate during cellular replication toward pure populations (homoplasmy) (19). When the mutation is a large deletion, the deleted molecules become enriched over time because replication rate is proportional to the length of the molecule and the smaller molecules replicate faster (3). Third, a cell will express a mtDNA mutant phenotype only when the extent of cellular energy deprivation falls below the minimum level required by that cell for normal function $(2,19)$.

Until recently, the diagnosis of the mitochondrial encephalomyopathies has relied on clinical, histochemical, and biochemical analysis $(5,20)$. However, the identification of molecular defects in these patients now makes molecular diagnosis feasible. The PCR technique (21) has made these tests preferable to more conventional approaches because it permits a molecular diagnosis from very limited specimen material. In this report, we will summarize current knowledge about mtDNA mutations associated with neuromuscular diseases and describe how the PCR permits rapid and accurate diagnosis of these complex diseases.

\section{MATERIALS AND METHODS}

$m t D N A$ extraction and $P C R$. mtDNA was enriched from fresh or frozen muscle as previously described (5). PCR was used to amplify normal and deleted mtDNA using synthetic oligonucleotides as primers (Microchemical Facility, Emory University). The $100-\mu \mathrm{L}$ PCR reactions contained $200 \mu \mathrm{M}$ of each dNTP (dATP, dTTP, dCTP, and dGTP), $50 \mathrm{mM} \mathrm{KCl}, 10 \mathrm{mM}$ Tris-Cl, $\mathrm{pH} 8.3,1.5 \mathrm{mM} \mathrm{MgCl}_{2}, 0.01 \%$ (wt/vol gelatin), $30 \mathrm{pmol}$ of each primer, and 2.5 U Taq polymerase (Perkins Elmer Cetus, Norwalk, CT). No oil overlay was used. When multiple primer pairs are included in a single reaction, $30 \mathrm{pmol}$ of each forward and reverse primer per target were used. PCR-generated DNA was visualized on agarose gels with ethidium bromide.

Sequencing. Asymmetrically PCR-amplified DNA for dideoxy sequencing was prepared by adding one primer in 100 -fold excess 
of the other (22). The thermal amplification profile, sequencing reaction mixtures, and PAGE protocols have been previously described (23). The procedure has been further modified by substituting 7-deaza-2'-deoxyguanosine-5' -triphosphate for deoxyguanosine- $5^{\prime}$-triphosphate in all nucleotide mixes to eliminate the $\mathrm{G}-\mathrm{C}$ compression common in mtDNA sequences.

\section{RESULTS}

LHON: PCR diagnosis of a specific base change. LHON is a maternally inherited disease (24) associated with late onset bilateral optic nerve death. During the acute phase, the optic nerve dies from the center outward, leading to central retinal scotoma. Before the acute phase, retinal examination reveals tortuous vessels and microangiopathy. Cardiac dysrhythmia is also a common finding, but muscle pathology has not been observed $(2,25,26)$.

We have identified the mtDNA mutation responsible for most cases of LHON. This mutation changes nt 11778 (27) from an A to a $\mathrm{G}$ and converts the 340th amino acid of NADH dehydrogenase from an arginine to a histidine. The mutation has been found in $80 \%$ of patients diagnosed with LHON, but is not found in the general population (6). Analysis of mtDNA haplotypes from American black and European white LHON patients has revealed that the mutation has occurred at least twice and in both cases resulted in the disease (7). The NADH dehydrogenase deficiency has been confirmed biochemically in one family (28). Thus, this mutation is a cause of the symptoms.

The LHON nucleotide substitution eliminates a SfaNI restriction site in the patient's mtDNA, providing a molecular diagnostic test for this disease. The sequence surrounding the mutation is amplified from blood cell DNA using primers at nt11673 $\rightarrow$ ) and $11875(\leftarrow)$ to yield a 212-bp fragment. Digestion of this fragment with SfaNI cuts the fragment into 117-bp and 95-bp fragments in control individuals but does not cut the fragment in patients with the mutation. The digestion products are readily resolved on NuSieve (FMC Bio Products, Rockland, $\mathrm{ME}$ ) agarose gels and detected by staining with ethidium bromide (Fig. 1) (6).

Ocular myopathies and other deletion syndromes: PCR diagnosis of deletions. The ocular myopathies are associated with chronic external ophthalmoplegia, ptosis, and RRF. More severe cases can also include neurosensory hearing loss, cerebellar ataxia, elevated cerebrospinal fluid lactate and protein, and cardiac dysrhythmias. The mild cases are described as CEOP, and the more severe are designated $\operatorname{KSS}(2,20)$.

mtDNA deletions associated with CEOP and KSS have been mapped to two sectors of the genome. Most deletions are located within the two-thirds of the genome encompassed by the $\mathrm{O}_{\mathrm{H}}$ and

\section{P P C C C}

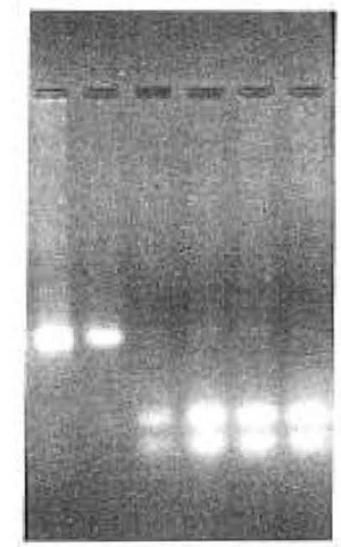

\section{$212 \mathrm{bp}$ \\ $117 \mathrm{bp}$ $95 \mathrm{bp}$}

Fig. 1. The 212-bp PCR-generated mtDNA fragment from LHON patients $(P)$ and normal controls $(C)$ digested with SfaNI and visualized with ethidium bromide on a $2.5 \%$ NuSieve, $0.9 \%$ Sea Kem agarose gel. the $\mathrm{O}_{\mathrm{L}}$, with the remainder in the third between the $\mathrm{O}_{\mathrm{L}}$ and the $\mathrm{O}_{\mathrm{H}}(3)$.

We have found that deletions in the two-thirds arc are associated with a 13-bp direct repeat (ACCTCCCTCACCA) at nt84708482 and nt13447-13459. This results in a 4977-bp deletion removing parts of ATPase 8 and ND5 and all of the intervening genes including ATPase 6, COIII, ND3, ND4L, and ND4. Numerous other direct repeats located in the two-thirds arc could account for most of the remaining mtDNA deletions through a slip replication (23) or homologous recombination (29).

Because these deletions appear as clonal, spontaneous mutations during somatic development, molecular analysis must be done on the affected tissue. For the ocular myopathies, this requires a muscle specimen, which can be reduced to a needle biopsy when using PCR. mtDNA deletions associated with other tissues, such as pancytopenia $(30,31)$, require the sample from the appropriate tissue.

Approximately $40 \%$ of mtDNA deletions are associated with the 13 -bp repeat $(14,29)$. Therefore, many optic myopathy patients can be diagnosed at the molecular level using PCR primers specific for this deletion. We can simultaneously amplify both the normal and deleted mtDNA using a forward primer at nt7392 $(\rightarrow)$ and two reverse primers at nt9244( $\leftarrow)$ and nt13950( $(\leftarrow)$.


Fig. 2. Upper panel: The forward $\rightarrow$ primer was located at nt73927410 and reverse $(\epsilon)$ primers were located at nt9244-9225 and nt 13950 13932 of the published human mtDNA sequence $(27)$. The nt7392 $\rightarrow$ ) and nt9244 $(\leftarrow)$ primer pair amplified a 1852-bp fragment from wild-type DNA template. The $n t 7392(\rightarrow)$ and $n t 13950(\leftrightarrow)$ primer pair amplified a 1581-bp fragment from the deleted mtDNA. Lower panel: PCR amplification products of muscle mtDNA, visualized with ethidium bromide. $P$, proband; $S 1$ and $S 2$, sisters of the proband; $S t d$, mol wt standards. The 1852-bp fragment is seen in all samples, whereas the 1581-bp fragment is only seen in the patient with the deletion (23). 
Primers at nt7392 $\rightarrow$ ) and nt9244( $\leftrightarrow$ ) amplify a 1.9-kb fragment from the normal mtDNA and primers nt7392 $\rightarrow$ ) and nt13950( () amplify at $1.6-\mathrm{kb}$ fragment from the deleted $\mathrm{mtDNA}$. The former acts as an internal control for the latter; the diagnosis is made if both fragments are observed on an ethidium bromide-stained agarose gel (23) (Fig. 2). Additional deletions in the two-thirds $\mathrm{O}_{\mathrm{H}}$-to- $\mathrm{O}_{\mathrm{L}}$ arc could be defined by combining several forward primers and/or reverse primers (32).

MERRF: PCR screening for intragenic deletions and point mutations. MERRF is a maternally inherited encephalomyopathy associated with uncontrolled myoclonus epilepsy and RRF $(5,20)$. We have characterized a large pedigree associated with MERRF and demonstrated that this pedigree has all of the characteristics expected for a heteroplasmic mtDNA mutation. First, the disease is maternally inherited with every individual along the maternal lineage being affected to some degree. Second, the severity of the symptoms along the maternal lineage varies from an individual with severe disease manifestations including myoclonus, dementia, hypoventilation, hearing loss, RRF, and severe electrophysiologic abnormalities of the CNS to an individual with only mild manifestations in the visual evoked response and EEG. Third, individuals along the maternal lineage have a biochemical defect in complexes I and IV of muscle and the severity of an individual's biochemical defect is directly proportional to the severity of his clinical manifestations (5).

Although MERRF patients have RRF, they are not associated with large deletions $(5,14)$. However, one patient with MERRF, who also displays the stroke-like symptoms of MELAS, had a $0.4-\mathrm{kb}$ deletion in the ND5 gene (15). Therefore, at least some MERRF and MELAS cases appear to result from mtDNA point mutations or small intragenic deletions.

To distinguish between these cases, we have prepared a series of PCR primers permitting amplification of all human mtDNA coding regions in nine fragments. The mtDNA fragments of the proband of our large maternally-inherited MERRF pedigree are shown in Figure 3. The right panel describes the fragments and indicates restriction endonucleases useful for further characterization. The left top panel shows the fragments resolved on an agarose gel and the left bottom panel shows their restriction

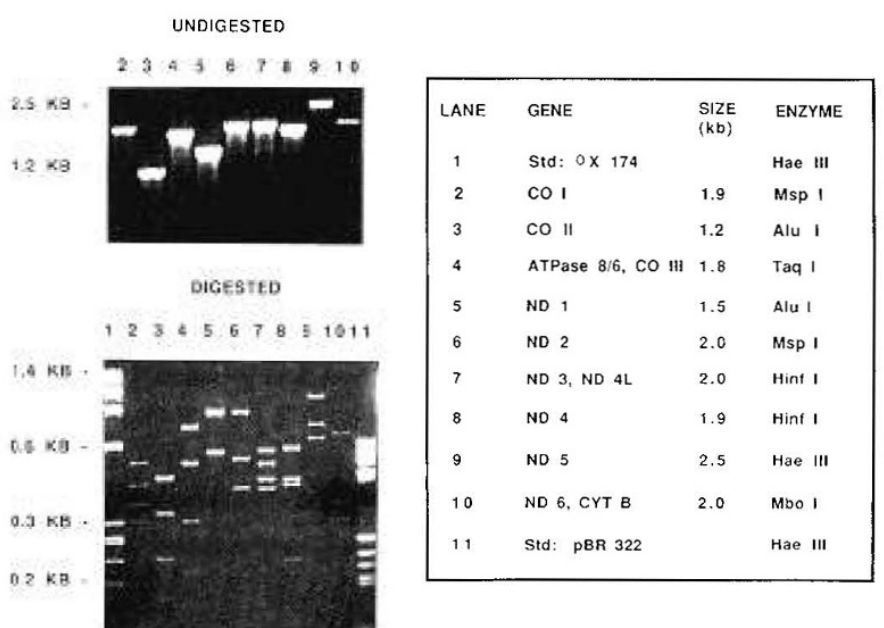

Fig. 3. Right panel: Identification of mtDNA regions amplified and restriction endonucleases used to subdivide them. Upper left photograph: Gel electrophoresis of PCR-amplified regions of mtDNA. PCR coordinates are: lane 2, nt5720-7608; lane 3, nt7392-8628; lane 4, nt828210107; lane 5, nt3007-4508; lane 6, nt3951-5917; lane 7, nt9911-11873; lane 8, nt10714-12576; lane 9, nt11711-14208; and lane 10, nt1391415865. All were run on a $1.5 \%$ Sea Kem agarose gel and stained with ethidium bromide. The lower left photograph shows the fragment pattern produced by digestion of the PCR-generated mtDNA fragments by the restriction endonucleases listed in the right panel. The digestion products were separated on a $2.5 \%$ NuSieve $/ 0.9 \%$ Sea Kem agarose gel.

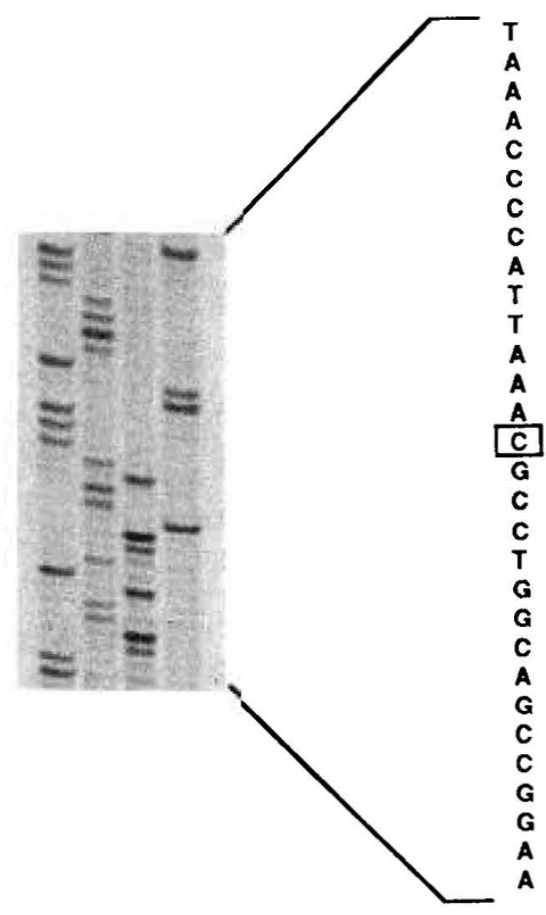

Fig. 4. Dideoxy sequencing using PCR-generated single-stranded mtDNA. The PCR amplification used primers at nt 12861 and nt 13950 in a ratio of 100:1. The boxed nucleotide reveals the deviation from the published sequence at nt 13702 .

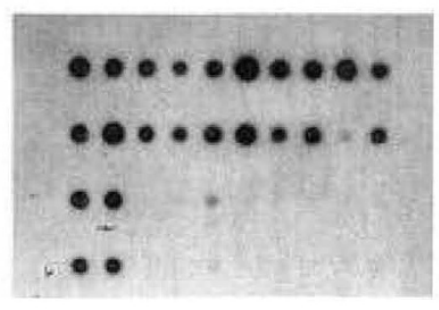

PANEL A

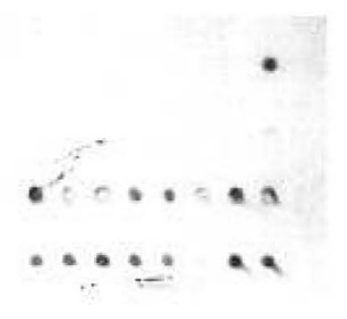

PANEL B
Fig. 5. Oligonucleotide hybridization of dot-blot filters to screen the mutation at nt8701. All samples were vacuum-blotted in duplicate amounts ranging between 10 and $20 \mathrm{ng}$ DNA. Panel $A$ : Filter probed with the nt8701 European white sequence (CAAATGATAaCCATACACA), hybridized at $35^{\circ} \mathrm{C}$, washed at $47^{\circ} \mathrm{C}$. This probe hybridized only to European samples. Panel $B$ : Duplicate filter probed with the nt8701 American black sequence (GTTTACTATcGGTATG), hybridized at $44^{\circ} \mathrm{C}$ and washed at $52^{\circ} \mathrm{C}$. This probe hybridized specifically to Mexican, Asian, and African samples (6).

fragments comparably resolved. If this patient were heteroplasmic for an intragenic deletion, then the PCR fragment encompassing that region would appear as a doublet. Such a deletion could be further mapped by identifying double bands in the restriction fragments of that amplified region. In our MERRF patient, we observed no heteroplasmic intragenic deletion. This was confirmed by densitometric analysis of the fragment mobility from the patient and by comparing the migration of his mtDNA fragments with those of a control.

This PCR experiment confirms our previous hypothesis that the mtDNA mutation of our MERRF pedigree (5) is a point mutation and not a deletion. It also demonstrates the utility of this method for mapping intragenic mtDNA deletions.

This analysis can be extended to the nucleotide level by direct sequencing of asymmetrically amplified PCR fragments. By this procedure, the mtDNA sequence can be rapidly screened for nucleotide substitutions as compared with available sequences 
from a European white (27) and an American black (6). A nucleotide substitution at nt13702 found in the MERRF proband is shown in Figure 4. This mutation changes a HaeIII restriction site, resulting in the fusion of two adjacent restriction fragments. In the lower right-hand panel of Figure 3, the top band in lane 9 is the altered fragment that migrates more slowly on the gel than predicted from the published sequence. This nucleotide substitution was found in all the mtDNA of patients and controls, both black and white (6), and is thus either a sequencing error or a common polymorphism.

During direct sequence analysis, nucleotide substitutions that alter amino acids become candidates for the disease-causing mutation. These can be tested to determine if they correlate with disease manifestations, either between pedigrees with the same symptoms, as for the homoplasmic mtDNA mutation of LHON, or between individuals in a pedigree, as for the heteroplasmic mtDNA mutation in MERRF. This screening is done using differential oligonucleotide hybridization to PCR-amplified mtDNA.

We observed an amino acid substitution in ATPase 6 that was a potential candidate for a MERRF mutation and which serves to illustrate the screening procedure. The nucleotide substitution is located at nt8701. An A encodes a threonine (ACC), and a $G$ encodes an alanine (GCC). The region surrounding this substitution was amplified using primers beginning at $n t 8557 \rightarrow)$ and $8929(\leftarrow)$. The resulting 373-bp fragment was alkaline denatured, transferred to nylon membrane, and duplicate filters hybridized to 19-base oligonucleotide probes homologous to the two sequences (6). Differential hybridization is shown in panels $A$ and $B$ of Figure 5. This analysis revealed that the sequence found in MERRF was an amino acid polymorphism specific to Europeans, not found in Asians and Africans. Therefore, this mutation is not the cause of MERRF.

\section{DISCUSSION}

Current procedures for the diagnosis of neuromuscular diseases resulting from mtDNA mutations require substantial muscle biopsies; frequently, the biochemical and histologic diagnoses are ambiguous. The identification of specific mtDNA molecular defects responsible for these symptoms now permit accurate diagnosis. The ability to amplify mtDNA from small samples using PCR permits molecular diagnosis using much smaller specimens than previously possible. It is now apparent that, for a number of mtDNA encephalomyopathies, PCR will become the preferred procedure in the years to come.

Note added in proof: Since this article was submitted, a mitochondrial point mutation at nt8334 (tRNA ${ }^{\text {Lys }}$ ) has been demonstrated to cause MERRF (Shoffner JM, Lott MT, Lezza AMS, Seibel P, Ballinger SW, Wallace DC 1990 Myoclonic epilepsy and ragged-red fiber disease (MERRF) is associated with a mitochondrial tRNA ${ }^{\mathrm{Lys}}$ mutation. Cell 61:931-937).

\section{REFERENCES}

1. Wallace DC 1986 Mitochondrial genes and disease. Hosp Pract [off] 12:41-49

2. Wallace DC 1987 Maternal genes: mitochondrial diseases. In: McKusick VA. Roderich TH. Mori J. Natalie WP (eds) Birth Defects. Alan R Liss, New York. pp 137-190

3. Wallace DC 1989 Mitochondrial DNA mutations and neuromuscular disease. Trends Genct 5:9-13

4. Wallace DC, Shoffner JM, Zheng X. Lott MT, Singh G. Krawiecki N 1989 Mitochondrial encephalomyopathies: diseases of two genomes. In: Stockdale F, Kedes L (eds) Cellular and Molecular Biology of Muscle Development, UCLA Symposia on Molecular and Cellular Biology, New Series. Alan R Liss, New York, pp 987-1009
5. Wallace DC. Zheng X. Lott MT, Shoffner JM. Hodge JA, Kelley RI, Epstein CM. Hopkins LC 1988 Familial mitochondrial encephalopathy (MERRF): genetic, pathophysiological, biochemical characterization of a mitochondrial disease. Cell 55:601-610

6. Wallace DC. Singh G. Lott MT, Hodge JA. Schurr TG, Lezza AMS, Elsas Il LJ. Nikoskelainen EK 1988 Mitochondrial DNA mutation associated with Leber's hereditary optic neuropathy. Science 242:1427-1430

7. Singh G, Lott MT, Wallace DC 1989 A mitochondrial DNA mutation as a cause of Leber's hereditary optic neuropathy. N Engl J Med 320:1300-1305

8. Holt IJ, Harding AE, Morgan-Hughes JA 1988 Deletions of mitochondrial DNA in patients with mitochondrial myopathies. Nature 331:717-719

9. Holt IJ, Cooper JM, Morgan-Hughes JA, Harding AE 1988 Deletions of muscle mitochondrial DNA. Lancet 1:1462.

10. Zeviani M, Moraes CT. DiMauro S, Nakase H, Bonilla E. Schon EA, Rowland LP 1988 Deletions of mitochondrial DNA in Kearns-Sayre syndrome. Neurology 38:1339-1346

11. Lestienne P, Ponsot G 1988 Kearns-Sayre syndrome with muscle mitochondrial DNA deletion. Lancet 1:885

12. Johns DR, Drachman DB. Hurko O 1989 Identical mitochondrial DNA deletion in blood and muscle. Lancet 1:393-394

13. Ozawa T, Yoneda M, Tanaka M, Ohno K. Sato W, Suzuki H, Nishikimi M, Yamamoto M. Nonaka I, Horai S 1988 Maternal inheritance of deleted mitochondrial DNA in a family with mitochondrial myopathy. Biochem Biophys Res Commun 154:1240-1247

14. Moraes C.T, DiMauro S, Zeviani M, Lombes A, Shanske S, Miranda AF, Nakase H. Bonilla E, Werneck LC, Servidei S, Nonaka I, Koga Y, Spiro AJ, Brownell AKW, Schmidt B. Schotland DL, Zupanc M, DeVivo DC, Schon EA, Rowland LP 1989 Mitochondrial DNA deletions in progressive external ophthalmoplegia and Kearns-Sayre syndrome. N Engl J Med 320:12931299

15. Saifuddin Noer A, Marzuki S, Trounce I, Byrne E 1988 Mitochondrial DNA deletion in encephalopathy. Lancet 2:1253-1254

16. Shuster RC, Rubenstein AF, Wallace DC 1988 Quantitation of mitochondrial DNA in anucleate human blood cells. Biochem Biophys Res Commun 155:1360-1365

17. Giles RE, Blanc H, Cann HM, Wallace DC 1980 Maternal inheritance of human mitochondrial DNA. Proc Natl Acad Sci USA 77:6715-6719

18. Case JT, Wallace DC 1981 Maternal inheritance of mitochondrial DNA polymorphisms in cultured human fibroblasts. Somatic Cell Mol Genet 7:103-108

19. Wallace DC 1986 Mitoic segregation of mitochondrial DNAs in human cell hybrids and the expression of chloramphenicol resistance. Somatic Cell Mol Genet 12:41-49

20. DiMauro S. Bonilla E. Zeviani M, Nakagawa M, DeVivo DC 1985 Mitochondrial myopathies. Ann Neurol 17:521-538

21. Saiki RK. Scharf S, Faloona F, Mullis KB, Horn GT, Erlich HA, Arnheim N 1985 Enzymatic amplifiction of $\beta$-globin genomic sequences and restriction site analysis for diagnosis of sickle cell anemia. Science 230:1350-1354

22. Innis MA. Myambo KB, Gelfand DH, Brow MA 1988 DNA sequencing with Thermus aquaticus DNA polymerase and direct sequencing of PCR-amplified DNA. Proc Natl Acad Sci USA 85:9436-9440

23. Shoffner JM. Lott MT, Voljavec AS. Soueidan SA. Costigan DA, Wallace DC 1989 Spontaneous Kearns-Sayre/chronic ophthalmoplegia plus syndrome associated with a mtDNA deletion: a slip-replication model and metabolic therapy. Proc Natl Acad Sci USA 85:7952-7956

24. Erickson RP 1972 Leber's optic atrophy, a possible example of maternal inheritance. Am J Hum Genet 24:348-349

25. Nikoskelainen E 1984 New apsects of the genetic, etiologic and clinical puzzle of Leber's disease. Neurology 34:1482-1484

26. Nikoskelainen EK, Savontaus M-L, Wanne OP. Katila MJ, Nummelin KU 1987 Leber's hereditary optic neuroretinopathy, a maternally inherited disease: a genealogic study in four pedigrees. Arch Ophthalmol 105:665-671

27. Anderson S. Bankier AT, Barrell BG, de Bruijn MHL, Coulson AR, Drouin J, Eperson IC, Nierlich DP, Roe BA, Sanger F, Schreier PH, Smith PH, Staden R. Young IG 198I Sequence and organization of the human mitochondrial genome. Nature 290:457-465

28. Parker WD. Oley CA. Parks JK 1989 A defect in mitochondrial electrontransport activity (NADH-coenzyme Q oxidoreductase) in Leber's hereditary optic neuropathy. N Engl J Med 320:1331-1333

29. Schon EA, Rizzuto R, Moraes CT, Nakase H. Zeviani M, DiMauro S 1989 A direct repeat is a hotspot for large-scale deletion of human mitochondrial DNA. Science 244:346-349

30. Rotig A, Colonna M, Blanche S, Fischer A, Le Deist F, Frezal J. Saudubray JM, Munnich A 1988 Deletion of blood mitochondrial DNA in pancytopenia. Lancet 2:567-568

31. Rotig A, Colonna M. Bonnefont JP. Blanche S. Fischer A, Saudubray JM, Munich A 1989 Mitochondrial DNA deletion in Pearson's marrow/pancreas syndrome. Lancet 1:902-903

32. Chamberlain J, Gibbs RA, Ranier JE, Nguyen PN, Caskey CT 1988 Deletion screening of the Duchenne muscular dystrophy locus via multiplex DNA amplification. Nucleic Acids Res 16:141-155 